includes primary care, acute, community services, public health, local authorities, the hospice and commissioners.

The group has developed into a highly-integrated strategic group that is able to facilitate and enact decisions and change based on the collective understanding and insight of the complex system. Rather than a group of individuals, this group now works and delivers as a strong and effective team. The effect of which is alignment and effectiveness in the system in a time of significant uncertainty and ambiguity.

Some key reasons for the success of this team is their focus on relationship and respect of and for all members, the lack of hierarchy and a collective focus, which is COVID-19.

During periods since March 2020 where COVID-19 was retreating, the group has considered 'wicked problems' within the local system, spending time considering urgent care, care homes and end-of-life and palliative care.

From the hospice's point of view, some of the key successes and contributions include:

- Offering to expand the catchment of the hospice rapid response service to provide additional overnight resource to district nursing.

- Seconding the hospice's rehabilitation team to provide additional community capacity where this was challenged.

- Being able to access COVID-19 vaccinations quickly and promptly by accessing vaccination capacity from partners from community services.

- Understanding pressures elsewhere in the system and better understanding system trends, giving the hospice insight into when demands on services are likely to peak.

- Enabled the hospice to tailor service provision to meet emerging needs, such as establishing a coordinated response to bereavement support with other local providers.

\section{P-213 A VISION FOR SYSTEM WIDE INTEGRATED PALLIATIVE AND END-OF-LIFE CARE}

${ }^{1}$ Wayne De Leeuw, ${ }^{2}$ Irene Watkins, ${ }^{3}$ Pippa Baker. 'Dorothy House Hospice, Winsley, UK; ${ }^{2}$ Prospect Hospice, Swindon, UK; ${ }^{3}$ Salisbury NHS Foundation Trust, Salisbury, UK

10.1136/spcare-2021-Hospice.228

Integrated Care Systems (ICSs) are central to the delivery of the NHS Long Term Plan and designed to bring together local organisations to redesign care and improve population health, creating shared leadership and action. In an ICS, NHS organisations, local authority and third sector partners such as hospices have the opportunity to 'take collective responsibility for the management of resources to improve the health of the population they serve'.

With these strategic opportunities on the horizon the BaNES, Swindon and Wiltshire (BSW) Palliative and End of Life Care Oversight Group, a combined provider and commissioner collaborative led by the three local hospice leads (Dorothy House, Prospect and Salisbury) formed in September 2020.

Over six months the group, made up of 30 provider organisations working in partnership with ICS commissioners, co-created a new strategy for palliative and end-of-life care based on:

1. National and local policy and strategy documents.

2. Quantitative analysis of local demographic and outcome data.
3. Qualitative/lived experience feedback obtained from an ongoing survey of patients', carers' and families' experiences of end-of-life care.

4. Mapping of services against items 1,2 and 3 above.

5. An ambition to deliver services that are value for money and delivering demonstrable positive outcomes for the local populace.

The strategy is framed by eight high level recommendations which highlight how success will be measured. The first, and arguably most innovative and/or controversial recommendation, is the creation of an ICS wide, hospice led, Palliative and End of Life Care 'Alliance'. With delegated budgetary authority this 'Alliance' will have accountability for ensuring equitable, outcome driven and cost efficient palliative and end-of-life care.

In June 2021 with unanimous ICS approval of the strategy, delegated transformation funds and hospice leadership, the 'Alliance' began meeting with an ambition to transform palliative and end-of-life care at scale across the system.

\section{P-214 STRATEGIC ALLIANCE TO INTEGRATE QUALITY DEMENTIA SERVICES ACROSS NORTH STAFFORDSHIRE}

Louise Eagle, Wendy Mountford. Douglas Macmillan Hospice, Stoke-on-Trent, Staffordshire

\subsection{6/spcare-2021-Hospice.229}

Background and Aims Approach Dementia Support and the Douglas Macmillan Hospice (Dougie Mac) joined together, in 2018, as a Strategic Alliance to ensure that local people affected by dementia are able to access the right support at the right time and live their life in the way that they choose, as dementia prevalence and impact increase nationally and locally.

Our vision is... to work together to integrate quality dementia services across our heartland of Stoke-on-Trent and North Staffordshire.

Our mission is... to respond effectively to the growing need for cohesive dementia support across our heartland by collaborating as two organisations with a shared ethos of compassion for the most vulnerable in our community.

Methods and anticipated Results To fulfil our mission and vision, we have prioritised the following strategic aims, which are to:

- Enable a consistent journey from dementia diagnosis to endof-life.

- Share resources as required to ensure the right support at the right time.

- Increase staff knowledge, skills and resilience as part of a team.

- Develop a carer enablement program from diagnosis to endof-life.

The introduction of a comprehensive assessment tool allows individuals, carers, Approach and Dougie Mac staff to assess the dementia stage of an individual, thus referrals can be made to the right services for their needs. One such service is the face-to-face support at the Dementia Services Centre, on-site at Dougie Mac, delivered in partnership by the Dementia Liaison Coordinator and Dementia Wellbeing Activity Officer to provide carer support in addition to an interactive stimulating activity session for the person living with dementia. 
Conclusion The Alliance will develop a joined-up, holistic model of dementia support for the local community which puts the individual at the centre of their support, thus providing an integrated dementia support offering for local people from diagnosis to end-of-life.

\section{P-215 MAKING AN IMPACT: TRANSFORMING PALLIATIVE AND END-OF-LIFE CARE IN LIVERPOOL}

${ }^{1}$ Carolyn Julie Bellieu, ${ }^{1}$ Kate Marley, ${ }^{2}$ Zoran Blackie, ${ }^{3}$ Helen BOnwick, ${ }^{3}$ Laura Chapman, ${ }^{3}$ Aileen Scott, ${ }^{3}$ Sarah Fradsham, ${ }^{3}$ Lynne Jones, ${ }^{1}$ Clare Forshaw, ${ }^{4}$ Kate Dreyer, ${ }^{1}$ Andrew Khodabukus, 'Sarika Hanchanale, 'Grace Ting, ${ }^{1}$ Amara Nwosu, ${ }^{1}$ Katherine Rugen, ${ }^{2}$ Patricia McGuinness, ${ }^{2}$ Paula Whitfield. 'Liverpool University Hospitals NHS Foundation Trust, Liverpool, UK; ${ }^{2}$ Merseycare NHS Foundation Trust, Liverpool, UK; ${ }^{3}$ Marie Curie Hospice, Liverpool, UK; ${ }^{4}$ Woodlands Hospice, Liverpool, UK

10.1136/spcare-2021-Hospice.230

Palliative and end-of-life care services across Liverpool and South Sefton have been transformed over the last year to ensure that patients and their families have access to the right care from the right people when needed via a single phone number.

Patients and their families struggled to navigate previously complex health care systems in Liverpool. There were many different teams providing care across the hospitals, hospices, and community, usually requiring a referral for each new encounter resulting in duplication of work, multiple handoffs between services, and confusion amongst referrers. This meant that there was inequity in the level of service patients received and some patients were unable to get the care they needed.

The IMPaCT (Integrated Mersey Palliative Care Team) model is a consultant-led service to support patients nearing the end-of-life which was developed by reorganising existing services with key stakeholder involvement in the design. The essence of its success is collaborative working between services to prevent crises and timely response to problems as they arise. There are regular multidisciplinary meetings to ensure that services are directed to the areas of most need and ensuring that patients do not 'slip through the net,' especially on discharge from hospital.

There were 1320 patients supported by IMPaCT in April 2021. Referrals and calls for help come from any healthcare professional or patients and families themselves. Each call or referral is handled by a nurse specialist from the hospital, hospice or community specialist palliative care teams based in one of the two hospice hubs who assesses the patient's needs and arranges intervention by the most appropriate person, removing the need for the patient to contact multiple agencies and reducing stress. The patient stays on the IMPaCT register unless they die, move away, or their illness is cured.

\section{P-216 AMBULATORY MEDICAL CARE: ACUTE PALLIATIVE CARE IN THE COMMUNITY}

Carolyn Julie Bellieu, Kate Marley, Clare Forshaw, Katherine Rugen. Liverpool University Hospitals NHS Foundation Trust, Liverpool, UK

10.1136/spcare-2021-Hospice.231
The IMPaCT (Integrated Mersey Palliative Care Team) model of care is designed to improve access for patients, their families, and professionals. The COVID-19 pandemic enabled implementation more quickly than envisaged.

Waiting times for medical appointments in our integrated palliative medicine service were in excess of two weeks, restricting the clinic system's ability to deal with acute problems. The team recognised that routine medical review of patients is often unnecessary, and with this in mind, all the referrals for outpatients are now assessed by a nurse specialist triage coordinator and those requiring routine follow-up are now fed into the nurse-led telephone assessment clinics for monitoring. There is a junior and a senior palliative medicine doctor assigned to the ambulatory medical clinic in the IMPaCT hub at Woodlands Hospice from Monday to Friday.

There are a small number of pre-planned appointment slots for follow up, but the rest are used for urgent assessment of patients in person, by video consultation or by phone. Domiciliary visits can be undertaken if needed on the day.

All admission requests and advice calls to the ward now come to the hub which means that the calls come into doctors with the necessary time to deal with issues as they arise, co-ordinating with other members of the team as needed. This also means the medical team on the inpatient unit have more time to see their patients. Co-location of the ambulatory medical clinics with the nurse triage coordinators has improved working relationships and means that all settings have an awareness of which patients are most complex. Admissions to the hospice are now appropriately more complex and the waiting time reduced to within 24 hours in the majority of cases in December 2020. Waiting times for medical assessments has reduced to within 24 hours of the request.

\section{P-217 PROMOTING COLLABORATIVE WORKING BETWEEN HOSPICE AND RESPIRATORY TEAMS TO IMPROVE PATIENT OUTCOME}

Fiona Pyrke, Amy Stoian. Greenwich and Bexley Community Hospice, London, UK

\subsection{6/spcare-2021-Hospice.232}

Background The Care Quality Commission states that the pandemic has highlighted the benefits of local health services collaborating and working in partnership (Care Quality Commission. Better care through collaboration, 2021). Collaboration can improve communication, reduce duplication of effort, improve working relationships and provide a better experience for health service users (Integrated Care and Support Pioneers Programme, 2014). With this in mind, we saw an opportunity for our hospice team to work in partnership with the community respiratory team, as historically there has been limited collaboration between the two.

Aims To improve and promote a respective understanding of each organisation's services. To promote the benefits of referring patients with advanced lung disease to the hospice at an earlier stage so they can fully benefit from our range of services.

Methods Two hospice physiotherapists were each seconded one day per week to the community respiratory team to assist patients with breathlessness management. The physiotherapists were allocated a caseload and from this they identified those patients who were currently also under the care of the hospice and those who could benefit from a referral. 